\title{
A Traffic-Aware Street Lighting Scheme for Smart Cities using Autonomous Networked Sensors
}

\author{
Sei Ping Lau, Geoff V. Merrett, Alex S. Weddell, Neil M. White
}

Electronics and Computer Science, University of Southampton, UK.

\begin{abstract}
Street lighting is a ubiquitous utility, but sustaining its operation presents a heavy financial and environmental burden. Many schemes have been proposed which selectively dim lights to improve energy efficiency, but little consideration has been given to the usefulness of the resultant street lighting system. This paper proposes a real-time adaptive lighting scheme, which detects the presence of vehicles and pedestrians and dynamically adjusts their brightness to the optimal level. This improves the energy efficiency of street lighting and its usefulness; a streetlight utility model is presented to evaluate this. The proposed scheme is simulated using an environment modelling a road network, its users, and a networked communication system - and considers a real streetlight topology from a residential area. The proposed scheme achieves similar or improved utility to existing schemes, while consuming as little as $1-2 \%$ of the energy required by conventional and state-of-the-art techniques.
\end{abstract}

Keywords: Adaptive street lighting, smart streetlights, smart cities, networked sensing

\section{Introduction}

With approximately 90 million streetlights installed worldwide, street lighting has become a ubiquitous utility that can be found in most urban areas [1]. Effective street lighting can reduce both crime and traffic collisions [2, 3], and encourage socio-economic activities at night. Several studies have shown that the installation of street lighting improves the perception of personal safety and security $[4,5]$. To realise a sustainable and liveable city, the concept of Smart Cities has been proposed. Key to this vision is that of a Smart Environment, requiring sustainable and efficient management of the environment and its limited natural resources through information and communication technology (ICT) [6]. Although the benefits of street lighting are clear, sustaining its operation has become a concerning issue to local governments, both financially and environmentally. Electric street lighting consumes $114 \mathrm{TWh}$ annually, leading to the emission of 64 million tonnes of $\mathrm{CO}_{2}$ [1]. With rapid urbanization, the financial and environmental burden of street lighting is expected to grow as the number of streetlights is predicted to increase by over $300 \%$ in the coming decade [7]. 
Conventionally, streetlights remain lit continually overnight. The start and end of this period is typically triggered by a clock with a predefined schedule, or an integrated light sensor indicating when the surrounding environment becomes dark. However, this conventional or 'always-on' lighting scheme can result in energy wastage, especially when street lighting is not required or full brightness is no longer necessary. Examples of this include the middle of the night when very low traffic volumes are expected. Thus, the use of time-based dimming approaches, such as Philips Chronosense and Dynadimmer [8], has been proposed whereby selected streetlights are completely switched off or dimmed at specific hours. Warwickshire County Council, UK, anticipate annual savings of $£ 0.5 \mathrm{~m}(\sim \$ 0.85 \mathrm{~m})$ and 3000 tonnes of carbon reduction if their streetlights are operated on this basis [9]. Although time-based dimming schemes show substantial savings both financially and environmentally, they overlook the original purpose of having street lighting. The reduction or suppression of street lighting during specific hours may severely impair road users' ability to navigate or to avoid obstacles [10], and lead to more accidents and crimes during these hours.

Recent advances in sensing and communication technologies have encouraged their adoption in streetlight control and monitoring, permitting fine-grained management of streetlight operation $[11,12,13]$. Remote- and sensor-controlled street lighting offers significant prospects for saving energy, as the continual adjustment of lighting levels is possible. In general, a remote control centre performs the necessary management and regulation of streetlight operation, such as dimming for energy conservation and monitoring the health of the streetlights. In some cases, streetlight operations are adaptively adjusted based on ambient information, such as weather and traffic conditions, collected by a local sensor array. Most of the proposed remote- and sensor-controlled street lighting schemes adopt long- and/or short-range wireless communication networks to establish a communication link between the remote control centre and an individual streetlight. Jing et al. [14] proposed the use of cellular networks and wireless sensor networks (WSNs) in their streetlight monitoring, control and diagnosis system. In their proposal, a centralised control centre governs the entire streetlight network, and a remote terminal unit (normally installed at the streetlight transformer stations) serves as a gateway between the centre and the sensor nodes. Cellular network based Internet connectivity is proposed to relay the commands and the status of the streetlights between the control centre and the gateways, and eventually propagate to the intended streetlights via the WSN. This use of communication networks can be found in many similar works $[11,12,13]$; however, many of these rely on a remote control centre that typically comes with a relatively high price tag [15]. Furthermore, they are also subject to a single point of failure as their performance depends upon the reliability of the long-range communication network.

For traffic-aware street lighting, a variety of different sensing mechanisms have been reported to allow autonomous adjustment of lighting level based on detected road users. Sun et al. [16] demonstrated the use of a multi-sensor module to prolong the operational hours of their standalone solar-powered streetlight. This module utilises a microphone and passive 
infrared sensor to allow detection of passing humans. A similar method was also adopted by many recent works [17, 18, 19, 20, 21], where a presence-detection module controlled the streetlights from a distance. Their sensors, however, had a limited sensing range which limited the effective range of their proposed method [22]. Instead of detecting the presence of road users, Müllner and Riener [23] proposed the use of a pedestrian tracking solution via a combination of Global Positioning System (GPS) and Internet-enabled smartphones. This combination allowed the system to track the precise location of a pedestrian, and hence fade-in and -out streetlights within a defined radius of them. Such usage of smartphones provides a potential mechanism for precise traffic-aware street lighting, but solutions are inherently limited to owners of such devices. This is illustrated by the fact that only $39 \%$ of people in the UK own smartphones, with a significant bias towards younger age groups (16 to 34 years old) [24]. Enabling GPS sensing and Internet connectivity in a smartphone has been shown to increase power consumption by $600 \mathrm{~mW}$ [25] and $650 \mathrm{~mW}$ [26] respectively. Considering a smartphone's limited battery capacity, such a power consumption can deplete a typical battery in a few hours. In addition, the issues surrounding location-privacy may also prove to be major obstacles of such traffic-aware street lighting schemes.

In recent years, many research projects have considered the use of ICT to allow precise and adaptive control of streetlights to conserve energy. This includes our early work in this field [27], where we proposed an algorithm which adjusted streetlight brightness in response to nearby road users, reducing the energy consumption by $30 \%$ compared to state-of-the-art schemes. Importantly, many of these existing works (including our own) were primarily aimed at reducing energy consumption, with very little consideration given to the associated impact of such schemes on the usefulness of the streetlights. More recently, we reported on StreetlightSim [28], a simulation environment that models both road traffic patterns and adaptive networked streetlights. To evaluate the usefulness of street lighting we also presented StreetlightSim's 'utility' model, which quantifies the utility, or usefulness, of street lighting from different road users' perspectives.

In this paper, we propose TALiSMaN, a distributed Traffic-Aware Lighting Scheme Management Network. We present a number of novel contributions over our previous work and the state-of-the-art:

- We propose a new adaptive street lighting algorithm (based on that we proposed in [27]), TALiSMaN, which tailors its operation to different road users. This is based on the utility model we presented in [28], and we present a more complete description of this here.

- Instead of requiring a centralised controller, TALiSMaN has been designed to operate autonomously over a shortrange mesh network (i.e. a network of decentralised and distributed networked streetlights).

- $\quad$ TALiSMaN is evaluated against existing and state-of-the-art techniques, through the simulation of a scenario using real traffic and geographical data. Results are analysed for both energy consumption and streetlight utility. This is the first work that has used this holistic approach to the evaluation of lighting schemes. 
Application-based simulations show that TALiSMaN is able to provide improved or comparable streetlight utility to existing street lighting schemes, but with a significant improvement in energy efficiency (requiring only 1-55\% of the energy, depending on traffic volume).

This paper is structured as follows: Section 2 presents the diverse requirements of street lighting from different road users' perspectives, and derives a model for streetlight utility. Subsequently, section 3 presents the details of TALiSMaN, which utilises WSNs to manage streetlights to minimise energy consumption while maximising utility. The proposed lighting scheme is evaluated by simulating a real streetlight topology from a residential area in Southampton, UK, and considers a range of different road traffic volumes. Section 4 details the evaluation scenario and parameters adopted in this paper, and section 5 presents the performance of the proposed lighting scheme in terms of the achieved streetlight utility and consumed energy.

\section{Quantifying the Utility of Street Lighting}

Effective street lighting enables road users to see more clearly, better and further for a variety of different activities. In some studies, the ergonomic comfort of lighting is also considered to address the psychological needs and well-being of its users [29]. To minimise the energy consumption of streetlights, the most straightforward option is to turn them off altogether (but this defeats their purpose); ideally, streetlights would only be turned on when they are useful. In this section, we consider what constitutes effective street lighting, from both a motorist's and pedestrian's perspective. These stakeholders are considered to be the major beneficiaries of street lighting. Subsequently, we detail a model (previously proposed in [28]) that quantifies the usefulness of street lighting, referred to as 'streetlight utility'.

\subsection{A Pedestrian's Perspective}

From a pedestrian's perspective, effective street lighting should assist them in obstacle avoidance and navigation, identification of other pedestrians (facial recognition), and make them feel safer [30, 31]. Obstacles can cause pedestrians to fall, potentially resulting in serious injuries. Thus, an effective street lighting scheme should improve their ability to detect obstacles in their path. As intuition would suggest, obstacle detection is improved as illuminance increases [32, 33]. However, pedestrians begin to avoid obstacles at a distance of between 6 to $7 \mathrm{~m}$, regardless of this [34]. Facial recognition of other pedestrians requires considerably higher illuminance compared to obstacle detection. It is reported that a streetlight at the minimum lighting level for a pedestrian should allow recognition of other road users at a distance of $4 \mathrm{~m}$ [30]. Nevertheless, pedestrians prefer to avoid collision with another at a distance of around 8 to $9 \mathrm{~m} \mathrm{[34].}$

We model the utility of street lighting for pedestrian obstacle detection, navigation and facial recognition by incorporating the aforementioned requirements. This is given by $U_{\text {ped (avoid) }}$, as shown in Eq. (1), where $\gamma(x, t)$ is the ratio 
of illuminance level at $x$ metres ahead of a pedestrian at time $t$ to the minimum required illuminance level for the road the pedestrian is travelling on. This assumes that the illumination within a $10 \mathrm{~m}$ segment of road is equally important for obstacle detection, navigation and facial recognition. In the UK, BS EN 13201-2 [35] outlines the minimum required illuminance levels for different residential roads which range from 2 to 15 lux. Generally, illuminance levels with lower values are adopted for quieter residential roads.

$$
U_{\text {ped(avoid })}(t)=\frac{1}{10} \int_{0}^{10} \gamma(x, t) d x
$$

Street lighting for pedestrians' perceived safety was studied by Haans and de Kort [36] using three different light distributions: conventional, ascending, and descending. In a conventional light distribution, all streetlights in the test location delivered the same illuminance. In a descending light distribution, the streetlights in the subjects' immediate vicinity delivered a higher illuminance, while those further away were incrementally lower. An ascending light distribution is the opposite of this. Based on their practical experiments, subjects expressed that they had a similar, and in some cases, better sense of safety while the streetlights were in a descending distribution. Among all the light distributions studied, the ascending light distribution was least favoured by subjects. Thus, streetlight utility for perceived safety, $U_{\text {ped }(\operatorname{prospect})}$, is given by:

$$
\begin{gathered}
U_{\text {ped(prospect })}(t)=\frac{1}{300} \int_{-150}^{150} z(x, t) d x \\
z(x, t)=\left\{\begin{array}{rc}
\varepsilon(x, t), & \varepsilon(x, t) \leq 1 \\
1, & \varepsilon(x, t)>1
\end{array}\right. \\
\varepsilon(x, t)=\frac{\gamma(x, t)}{1-0.2\left\lfloor\frac{|x|}{30}\right\rfloor},-150 \leq x \leq 150
\end{gathered}
$$

where $x$ is the distance in metres from a pedestrian at time $t, z(x, t)$ is the ratio of illuminance level at location $x$ metres from a pedestrian at time $t$ to the illuminance level required at illumination zone where location $x$ is located.

In this model, $U_{\text {ped(prospect) }}$ considers a lit road segment $150 \mathrm{~m}$ before and after a pedestrian. These road segments are subdivided into five illumination zones (each segment covers a $30 \mathrm{~m}$ length of road, the typical coverage of a single streetlight) where each zone requires different minimum illuminance levels. The minimum illuminance of the nearest zones are at $100 \%$ according to the minimum required illuminance level for the road the pedestrian is travelling on. For each zone further away from the pedestrian, their minimum illuminance values are gradually decreased by $20 \%$. The road segments that are outside these illuminance zones are not required to be lit. 
Over a period of time, a pedestrian will perform both obstacle detection and navigation/awareness. Hence, the overall streetlight utility for pedestrian is given by:

$$
U_{\text {ped }}(t)=\alpha U_{\text {ped }(\text { avoid })}(t)+(1-\alpha) U_{\text {ped }(\text { prospect })}(t)
$$

where $\alpha$ is the weight of the time spent by pedestrian looking at the footpath. In this paper, we adopt a value of $\alpha=0.45$. This is based on the findings of Davoudian and Raynham [31], who found that pedestrians spend $40-50 \%$ of their time looking at the pavement.

\subsection{A Motorist's Perspective}

From a motorist's perspective, street lighting helps to extend and broaden their visual range beyond that offered by vehicle headlamps. This allows them to detect potential hazards in their direction of travel. In general, an effective street lighting scheme considers the average luminance, luminance pattern (also known as uniformity), threshold increment and surround ratio [30]. Among these factors, average luminance and uniformity affect motorists' ability to detect potential hazards. The effect of various illuminances and uniformities on hazard detection has been extensively studied [37, 38, 39, 40]. These studies report that motorists perform better at hazard detection when luminance and uniformity are increased. Hazard detection is normally associated with the proximity of the hazard to the vehicle in either time or in distance, in which appropriate manoeuvres can be carried out to avoid a collision and thus reduce the probability of injury to oneself or other road users. This distance is reflected as the stopping distance where a motorist must be able to stop their vehicle safely after a potential hazard is detected [41]. Usually, the stopping distance is between $60 \mathrm{~m}$ to $160 \mathrm{~m}$ based on factors including the vehicle's speed, road surface conditions, and the motorist's reaction time.

To model the streetlight utility for a motorist, we consider that they require a segment of road ahead of them to be illuminated, hence allowing them to detect potential hazards within their travelling path and bring their vehicle to a stop. As this road segment is typically between $60 \mathrm{~m}$ to $160 \mathrm{~m}$, a value of $100 \mathrm{~m}$ [41] is adopted in our model as it includes most of the stopping distances required in a residential road. In the UK, residential roads typically have a speed limit of $30 \mathrm{mph}$ (approximately $15 \mathrm{~m} / \mathrm{s}$ or $50 \mathrm{~km} / \mathrm{h}$ ). Assuming that the illuminance level within this road segment is equally important, and this road segment is lit at the minimum required illuminance level for the road a motorist is travelling on, the streetlight utility for a motorist, $U_{m o t}$, is modelled by:

$$
U_{m o t}(t)=\frac{1}{100} \int_{0}^{100} \gamma(x, t) d x
$$

where $\gamma(x, t)$ is the ratio of illuminance level at $x$ metres ahead of a motorist at time $t$ to the minimum required illuminance level for the road where the motorist is travelling on. 
In the next section, these utility models are considered in the design of a traffic-aware adaptive lighting scheme. This scheme aims to maximise the utility of the streetlights, and minimise their energy use.

\section{A Traffic-Aware Lighting Scheme Management Network (TALiSMaN)}

Based on the proposed utility models, two observations can be reached: (1) the lengths of the required lit road segments are finite, i.e. $150 \mathrm{~m}$ and $100 \mathrm{~m}$ for pedestrians and motorists respectively; and (2) within these required lit road segments, pedestrians and motorists require different light patterns. TALiSMaN exploits these properties by progressively adjusting the illuminance of streetlights according to different road users' needs, and improves their energy efficiency by minimising their energy use. TALiSMaN detects road users and shares the information with nearby streetlights. Upon receiving the information, they cooperate to create optimum lighting conditions that meet the road users' needs, and avoid illuminating the road at higher levels as this simply wastes energy.

The different user needs for street lighting and the proposed utility models in section 2 were considered, and translated to a relationship between a streetlight's proximity to the road user and the desired illuminance. This is summarised in Table 1. Instead of relying on a remote centralised control centre for managing streetlight operation, we propose to implement TALiSMaN over an autonomous WSN. Each streetlight incorporates a wireless sensor node with a short-range wireless communication module. This allows it to form a multi-hop WSN with neighbouring streetlights to exchange information. The network is time-synchronized, and each streetlight is pre-programmed with its own location information and unique identification, which are shared with others during network setup.

\section{Table 1}

The relationship between road users' distance and streetlight illuminance output.

\begin{tabular}{lcc}
\hline Road User Type & Distance from streetlight, & Illuminance output \\
\hline & $0 \leq d<30$ & $100 \%$ \\
Pedestrian & $30 \leq d<60$ & $80 \%$ \\
& $60 \leq d<90$ & $60 \%$ \\
& $90 \leq d<120$ & $40 \%$ \\
Motorist & $120 \leq d \leq 150$ & $20 \%$ \\
& $0 \leq d \leq 100$ & $0 \%$ \\
\hline
\end{tabular}

The streetlights are equipped with a light controller and a road-user sensor $[16,22]$. The light controller modulates the lamp output to switch the streetlight on, off, or adjust its illuminance. To allow near-instant response to continually changing illuminance requests, it is assumed that each streetlight uses a dimmable light-emitting diode (LED) lamp, and that its beam pattern covers the limited area of a single road segment. Although these sensor-based methods provide less 
accuracy in detecting road-user positions than GPS-based systems [23], they do not require the road-users to be instrumented with dedicated smartphone apps or other hardware, while still providing a substantial energy saving.

The following subsections explain the operation and implementation of TALiSMaN.

\subsection{System Operation}

In order to enable progressive control of the streetlight illuminance, based on the either presence detected by local sensors or information relayed by neighbouring nodes, four different operational states are defined in TALiSMaN: 'Lamp on by sensor', 'Lamp on by neighbour', 'Lamp on by delay' and 'Lamp off'. Amongst these operation states, 'Lamp off' and 'Lamp on by sensor' are shared between neighbouring sensor nodes using the on-board wireless communication module. Figure 1 shows the state machine of these operation states during operational hours.

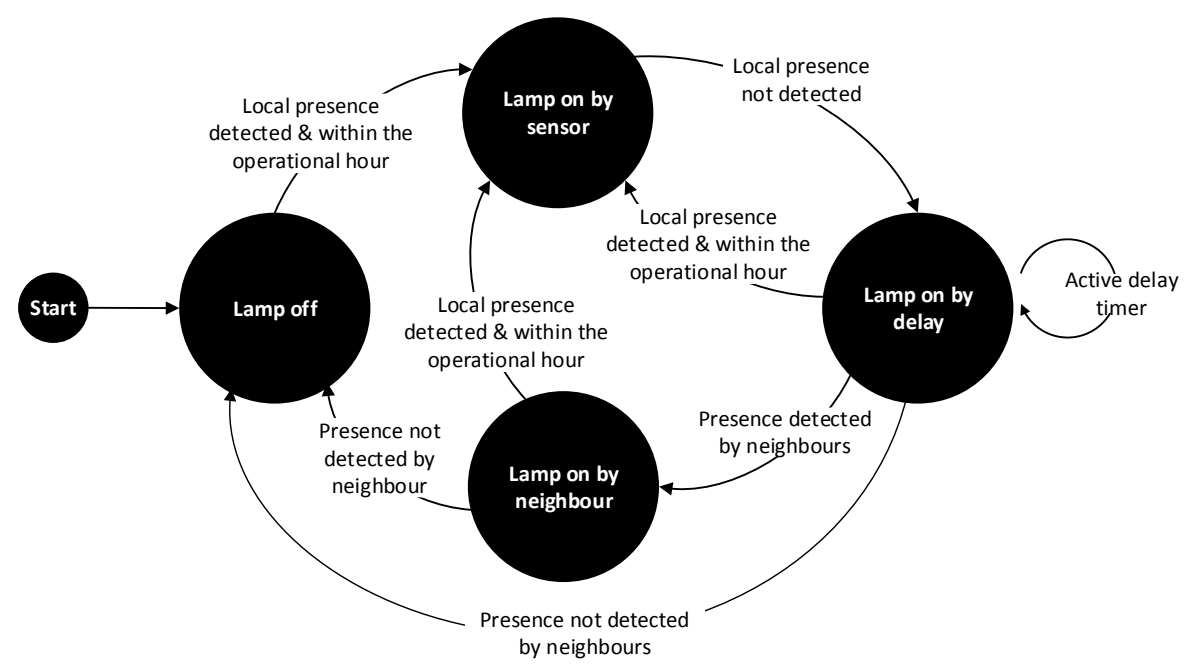

Figure 1. TALiSMaN operation state machine during streetlight operational hours.

When TALiSMaN shifts to 'Lamp on by delay', the state delay counter is reset and activated (see section 3.3 for state delay counter). The operation state will remain here until the state delay counter expires or a road user is detected by a local road-user sensor. Once the state delay counter expires, the received neighbours' operation states are evaluated, and operation state shifts to 'Lamp on by neighbour' only if neighbours are on. At this state, the streetlight remains switched on and its illuminance is adjusted to deliver the required lighting pattern (see section 3.2 for detail). The streetlight is switched off if none of the local or the neighbouring road-user sensors have detected the presence of any road users. While the operation state is 'Lamp on by neighbour' or 'Lamp off', the operation states from neighbouring nodes are evaluated as each packet is received. 


\subsection{Adjusting the Illuminance}

For TALiSMaN, the illuminance of the streetlights is adjusted for energy conservation while maintaining the optimum usefulness of having street lighting. However, creating the lit road segment that satisfies different road users' needs requires coordination between several streetlights. This is due to the assumptions that a road-user sensor has a limited detection range and the coverage from the streetlight beam pattern is finite. The coordination between streetlights is facilitated by sharing the road users' presence information with other sensor nodes within the required lit road segments. By sharing this information, the relative distance to the detected road users can be approximated using the Euclidean distance to the nearest sensor node. While the precise location of a road user is unknown, the receiving nodes assume that the detected road users are at the 'best-case' distance from them. This approximate relative distance, $d_{\text {aprox }}$ assumes that the road user is always located at the nearest edge of the sensor range, see Figure 2 and Figure 3.

The following sections detail the modulation of streetlight illuminance output upon detection of a pedestrian and a motorist.

\subsubsection{Illuminance Modulation based on Detected Pedestrians}

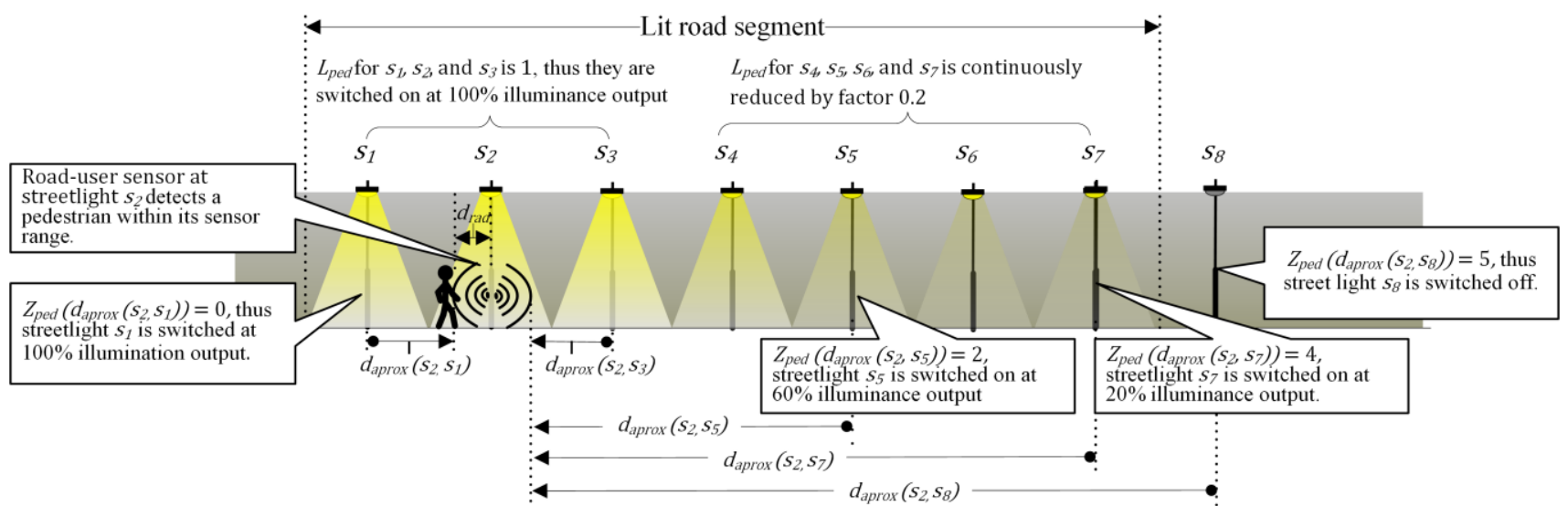

Figure 2. A lit road segment created by streetlight $s_{1}$ to $s_{7}$ while operating using the TALiSMaN. This lighting pattern is created based on the approximate relative distance to the detected pedestrian.

Figure 2 shows the development of the required lighting pattern after a pedestrian is detected by a road-user sensor at streetlight $s_{2}$. After the presence of the pedestrian is shared amongst the sensor nodes at streetlight $s_{1}$ to $s_{8}$, the illuminance of the respective streetlights, $L_{\text {ped }}$ is progressively modulated according to the following algorithm:

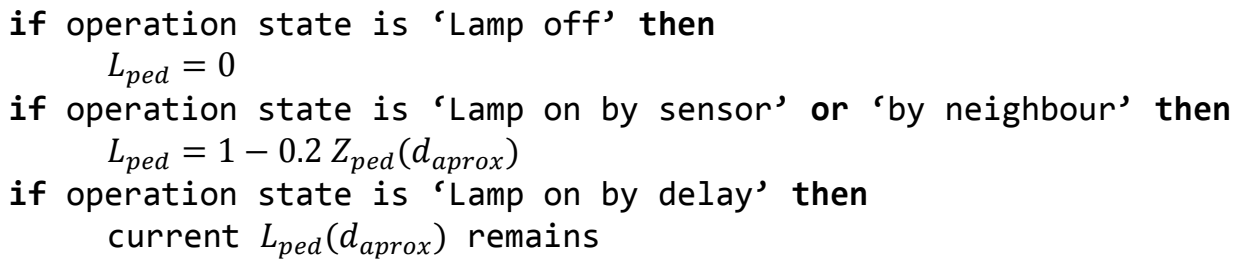


where $L_{p e d}$ is the required illuminance of the streetlight based on the approximate relative distance $(\mathrm{m})$ to the detected pedestrian, and $d_{\text {aprox }}$ and $Z_{\text {ped }}$ determines the illumination zone of the streetlight according to $d_{\text {aprox }}$.

In section 2.1, five different illumination zones were considered for pedestrians, and the required illuminance of each zone sequentially reduced by $20 \%$. To allow streetlights in different illumination zones to operate at these required illuminance levels, $L_{p e d}$ is sequentially reduced by a factor of 0.2 per zone. Since each illumination zone is $30 \mathrm{~m}$ in length and a pedestrian requires a lit road segment of $150 \mathrm{~m}$ before and after them, $Z_{\text {ped }}$ has maximum value of five. The illumination zone of a streetlight, $Z_{\text {ped }}$ with respect to $d_{\text {aprox }}$ is given by:

$$
\begin{gathered}
z_{\text {ped }}\left(d_{\text {aprox }}\right)=\left\{\begin{aligned}
0, & \left\lfloor\frac{d_{\text {aprox }}}{30}\right\rfloor=0 \\
\left\lfloor\frac{d_{\text {aprox }}}{30}\right\rfloor-1, & 0<\left\lfloor\frac{d_{\text {aprox }}}{30}\right\rfloor \leq 5 \\
5, & \left\lfloor\frac{d_{\text {aprox }}}{30}\right\rfloor>5
\end{aligned}\right. \\
d_{\text {aprox }}=\left\{\begin{aligned}
0, & d_{\text {rad }} \geq d_{\text {det }} \\
d_{\text {det }}-d_{\text {rad }}, & d_{\text {rad }}<d_{\text {det }}
\end{aligned}\right.
\end{gathered}
$$

where $d_{d e t}$ is the Euclidean distance (m) to the nearest sensor node that detects the pedestrian and $d_{\text {rad }}$ is the maximum detection range $(\mathrm{m})$ of the road-user sensor.

\subsubsection{Illuminance Modulation based on Detected Motorists}

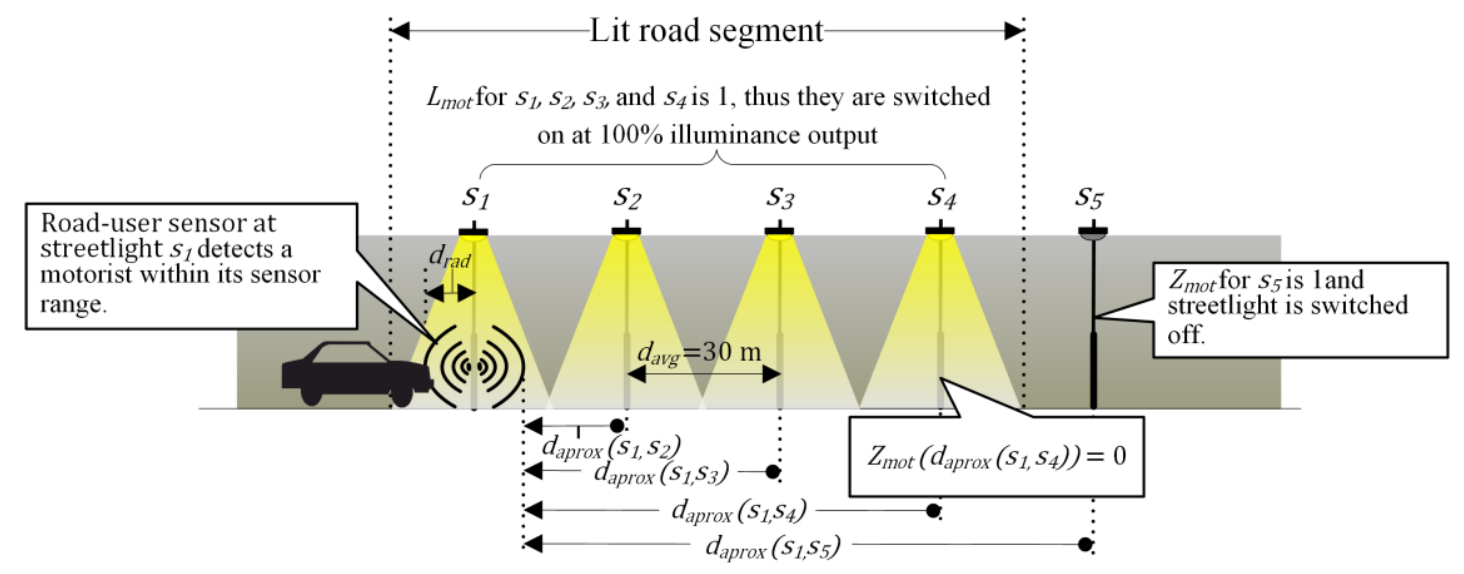

Figure 3. A lit road segment created by streetlight $s_{1}$ to $s_{4}$ while operating TALiSMaN. This lighting pattern is created based on the approximate relative distance to the detected motorist.

As the difficulty in detecting potential hazards increases with darker lighting conditions [10], the streetlight illuminance required by a motorist, $L_{m o t}$ is always at $100 \%$ for a road segment $100 \mathrm{~m}$ ahead of them. Since the road-user 
sensors are assumed to be unable to detect the direction that the detected motorist is travelling in, both road segments before and after the motorist are lit. Figure 3 illustrates the development of the required lighting pattern upon detection of a motorist by a road-user sensor at streetlight $s_{1}$. After the presence of the motorist is shared between streetlights $s_{1}$ to $S_{5}$, their illuminance is modulated according to the following algorithm:

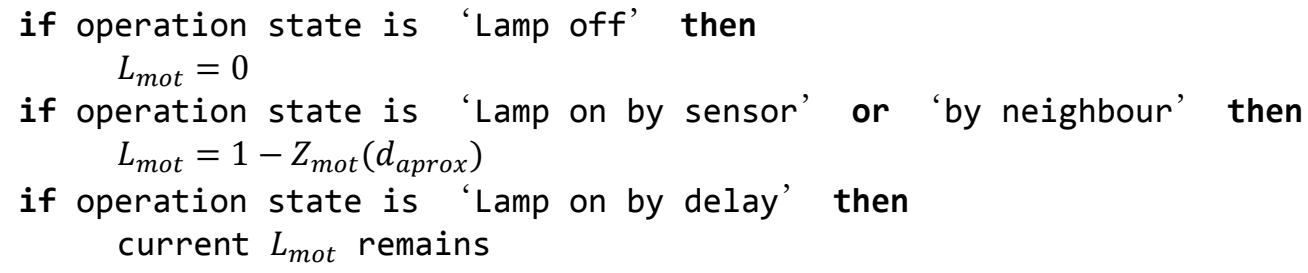

where $Z_{m o t}$ is the function that determines whether a streetlight is within the required road segment need to be lit. $Z_{m o t}$ is given by:

$$
Z_{\text {mot }}\left(d_{\text {aprox }}\right)= \begin{cases}0, & \left.\mid \frac{d_{\text {aprox }}}{d_{\text {avg }}}\right\rfloor \leq\left\lceil\frac{100}{d_{\text {avg }}}\right\rceil \\ 1, & \left.\mid \frac{d_{\text {aprox }}}{d_{\text {avg }}}\right\rfloor>\left\lceil\frac{100}{d_{\text {avg }}}\right\rceil\end{cases}
$$

where $d_{\text {aprox }}$ is the approximate relative distance $(\mathrm{m})$ to the detected motorist (see Eq. (8)), and $d_{\text {avg }}$ is the average distance to the next adjacent streetlight (assumed to be $30 \mathrm{~m}$ ).

\subsubsection{Combined Pedestrian-Motorist Illuminance Modulation}

Whenever pedestrians and motorists are detected simultaneously, the illuminance output of the streetlight is set to $\max \left(L_{\text {ped }}, L_{\text {mot }}\right)$. This is to ensure that an optimum lit environment can be provided both to pedestrians and motorists.

\subsection{State Delay Counter}

Void regions result from gaps in sensor coverage between streetlights. When road users travel into them, they can potentially cause unnecessary adjustment of streetlight illuminance. To reduce the impact of void regions, a state delay counter is adopted to prolong the TALiSMaN operation state at 'Lamp on by delay' until the road user is believed to have left the void region and entered the sensing range of the next streetlight. This feature also mitigates the latency of the communication network, which is particularly relevant when neighbouring sensor nodes detect the presence of road users simultaneously and compete for the communication channel to disseminate the information.

In this paper, the expiration time of the state delay counter, $t_{\text {exp }}$, is given by:

$$
t_{\text {exp }}=\frac{d_{a d j}-2 d_{r a d}}{v}, \quad d_{a d j} \geq d_{\text {rad }}
$$


where $d_{a d j}$ is the distance $(\mathrm{m})$ to the furthest adjacent streetlight, $d_{\text {rad }}$ is the detection range $(\mathrm{m})$ of the sensor node and it is assumed to be $13 \mathrm{~m}[22]$ and $v$ is the expected slowest travelling speed $(\mathrm{m} / \mathrm{s})$ of a particular road user.

Considering that only the presence of the road users is known, we use the road user's slowest travelling speed to compute $t_{\text {exp }}$. We assume that the slowest travelling speed of a motorist on residential roads is $4.5 \mathrm{~m} / \mathrm{s}$ ( 10 miles per hour). For pedestrians, $0.73 \mathrm{~m} / \mathrm{s}$ is assumed to be the slowest walking speed after considering the $5^{\text {th }}$ percentile of the pedestrian walking speed distribution (mean walking speed is $1.34 \mathrm{~m} / \mathrm{s}$ and standard deviation is $0.37 \mathrm{~m} / \mathrm{s}$ ) [42]. If both motorists and pedestrians are detected at any one moment, the pedestrian's slowest walking speed is always used to compute $t_{\text {exp }}$.

\section{Simulation Setup}

To evaluate the efficiency and effectiveness of TALiSMaN, it requires the modelling of vehicles, road networks, algorithms, and communication systems. However, existing tools were not available for this. Therefore, StreetlightSim [28], a simulation environment combining the OMNeT++ and SUMO simulation tools was created to model both traffic patterns and networked streetlights. This environment is used in this paper to evaluate the performance of TALiSMaN against existing works. StreetlightSim is open-source and freely available to the community [43]. Figure 4 (a) shows StreetlightSim simulating 112 streetlights based on the topology shown in Figure 4 (b). As illustrated by Figure 4 (a), only those within the required lit road segments are switched on (shown by white dots) upon detection of road users. Each lighting scheme is simulated over ten repeated simulation runs for every combination of traffic patterns and traffic volumes specified in section 4.2 .

The following subsections detail the simulation scenario and parameters adopted in this paper.

\subsection{Streetlights}

An actual streetlight topology located in a residential area was modelled for this evaluation. The locations of these streetlights were identified using an aerial photograph. Figure 4 shows the topology of the streetlights (represented by dots) in a residential area in Southampton, UK. In total, there are 112 streetlights placed over approximately $3.5 \mathrm{~km}$ of residential roads. For the purposes of evaluation, each streetlight is assumed to be equipped with a 25 W LED lamp which can illuminate a $30 \mathrm{~m}$ road segment. The streetlights are assumed to start operation at sunset and finish at sunrise the next day. Based on these observations, the distance between streetlights varies - for example as a result of roundabouts and junctions. Thus, a near-optimal streetlight utility is achieved, as shown in Figure 8.

The energy consumption of the different lighting schemes is influenced by the duration of their operation. This is dependent upon the geographical location, season, weather, and local environment. Therefore, our simulation scenario 
limits operational hours from 16:00 to 08:00 (16 hours) for clarity of evaluation. These operational hours represent one of the longest streetlight durations required during winter months in the UK.

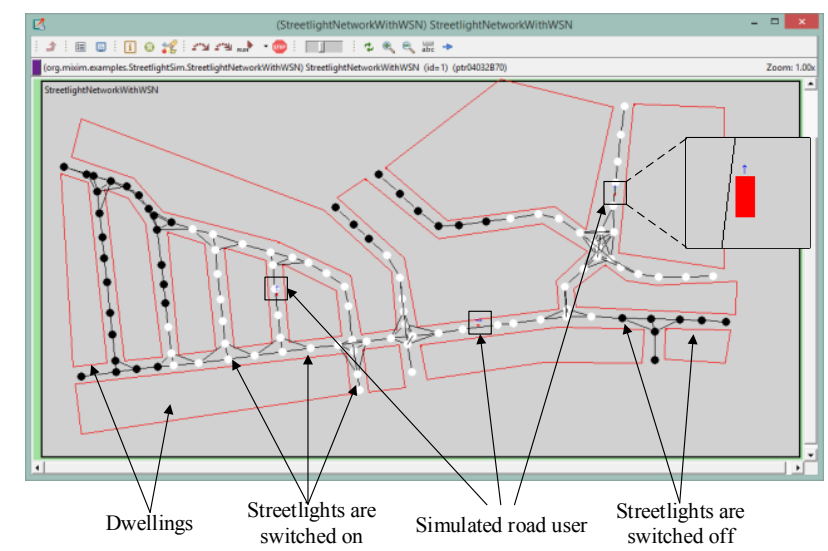

(a)

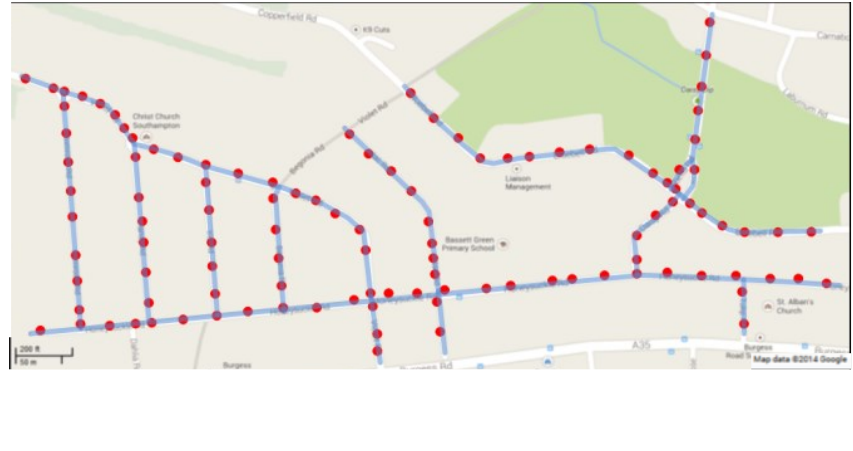

(b)

Figure 4. A Street lighting topology that consists of 112 streetlights scattered within $3.5 \mathrm{~km}$ of residential roads. (a) Snapshot of StreetlightSim simulating the TALiSMaN lighting scheme with the topology. White dots represent streetlights switched on due to their distance from detected road users, whereas black dots represent streetlights that are not within the required lit road segments, and thus they are switched off. (b) The locations of the streetlights (dots) and road network (shaded lines) considered during the simulations (the base map was adapted from Google Maps).

\subsection{Road Traffic}

To approximate the volume of actual road traffic in the residential roads (represented by shaded lines in Figure 4(b)), five different daily traffic volumes are considered during simulations. These values are 180, 438, 1347, 3508 and 6554 vehicles per day which represent the minimum, $1^{\text {st }}$ quartile, median, $3^{\text {rd }}$ quartile and maximum 'annual average daily traffic flow' (AADF) values respectively for residential roads in Southampton, UK [44]. Since AADF only accounts for vehicular traffic, an additional $14 \%$ is added to these values to represent pedestrian traffic; this value is based on data supplied by Southampton City Council [45]. Two traffic profiles, namely weekday and weekend, are considered during simulation as they demonstrate distinct traffic trends, see Figure 5. The mobility of a simulated pedestrian and a simulated motorist is governed by a total of 100 random routes. The mobility speed of these road users varies according on route traffic conditions but is limited to maximum $1.9 \mathrm{~m} / \mathrm{s}$ for a pedestrian [42] and $30 \mathrm{mph}$ for a motorist (the speed limit on residential roads in the UK). Detailed information on generating and injecting road traffic during simulations can be found in our previous work [28]. 


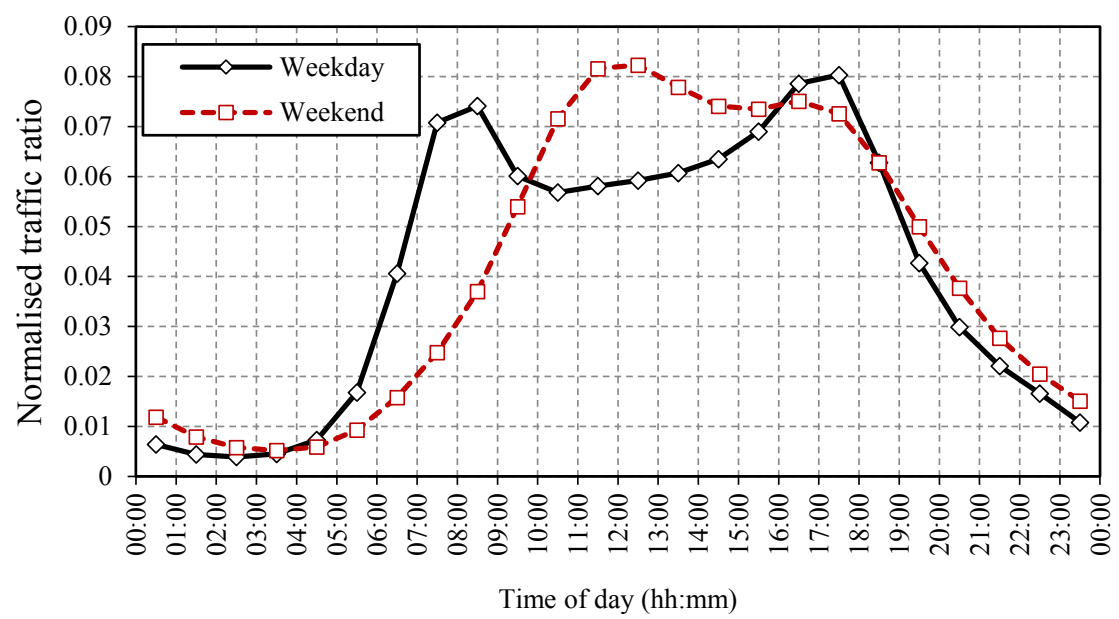

Figure 5. The traffic distribution ratio by time for the average weekend and weekday traffic (adapted from [46]).

\subsection{Baseline Lighting Schemes}

A range of different approaches have been proposed to reduce the energy consumption of street lighting, as outlined in Section 1. In our evaluation, we evaluate and compare TALiSMaN against the state-of-the-art approaches listed in Table

2. The Conventional (or 'always-on') lighting scheme is also included in our evaluation to serve as a benchmark for streetlight utility.

Table 2

Summary of streetlight schemes evaluated in this paper.

\section{Lighting Scheme}

Operation

\begin{tabular}{ll}
\hline $\begin{array}{l}\text { Conventional / always-on } \\
\text { Philips Chronosense [8] }\end{array}$ & $\begin{array}{l}\text { All the streetlights are switched on with 100\% illuminance output during the simulations. } \\
\text { Similar to Conventional lighting scheme except from 22:00 until 05:00 when illuminance } \\
\text { output of all the streetlights is reduced to 65\%. }\end{array}$ \\
Part-night [9] & $\begin{array}{l}\text { All the streetlights are switched on at 100\% except from 00:00 to 05:30 where the streetlights } \\
\text { are switched off completely to conserve energy. }\end{array}$ \\
Philips Dynadimmer [8] & $\begin{array}{l}\text { Streetlights operate at 40\% between 23:00 and 05:00, 55\% from 05:00 to 06:00 and } 65 \% \text { from } \\
\text { 20:00 to 23:00. For the rest of the operational hours, all the streetlights operate at } 90 \% .\end{array}$ \\
Multi-sensor [22] & $\begin{array}{l}\text { The streetlights are always switched on at } 40 \% \text {, and increased to 70\% and } 100 \% \text { if the distance } \\
\text { between the streetlight and road user is } 20 \text { m and } 10 \text { m respectively. }\end{array}$ \\
& $\begin{array}{l}\text { This lighting scheme assumes that the pedestrians are tracked via GPS- and Internet-enabled } \\
\text { smartphones. The streetlights within the user-defined radius are switched on at } 100 \% \text { and } \\
\text { those beyond the defined radius are switched off completely. For comparison purposes, the } \\
\text { radius of } 150 \mathrm{~m} \text { is considered during the simulations. Motorists are not tracked by the scheme, } \\
\text { but gain some consequential benefit from lighting intended for the pedestrians. }\end{array}$
\end{tabular}




\subsection{Wireless Sensor Network}

As discussed in section 3, the illuminance of the streetlights is individually controlled by a wireless sensor node, based on the presence of road users. To enable the effective detection of road users, these nodes are assumed to be equipped with a multi-sensor array. Details of the sensor array, however, are outside the scope of this research. The sensing range and sample rate vary considerably between technologies [22]. For the purposes of evaluation, Table 3 summarises the parameters that are adopted in our simulations.

\section{Table 3}

Sensor parameters and values.

\begin{tabular}{ll}
\hline Parameter & Value \\
\hline Sensing range & $13 \mathrm{~m}[22]$ \\
Sensor sampling rate & $20 \mathrm{~Hz}$ \\
\hline
\end{tabular}

Information on detected road users is time-sensitive, so it has to be relayed to neighbouring sensor nodes with minimal delay. If this information is relayed too late, the performance of TALiSMaN will be reduced, because streetlights will respond less quickly to road users' movements, reducing utility and/or increasing energy consumption. To address this need, we assume that all the sensor nodes operate using an IEEE 802.15.4 non-beacon enabled mode and adopt carrier sense multiple access (CSMA) with collision avoidance as their media access control layer protocol [47]. While operating in this mode, sensor nodes are always active and ready to relay any information as required. Table 4 summarises the communication parameters adopted during the simulations. Since the focus of this work is on the energy efficiency of street lighting schemes and their respective streetlight utility, the simple path loss radio propagation model from the MiXiM framework was used in this work. To account for random variation in the channel, log-normal shadowing is also applied.

\section{Table 4}

IEEE 802.15.4 parameters and their values.

\begin{tabular}{ll}
\hline Parameter & Value \\
\hline Bit rate & $250 \mathrm{kbps}$ \\
Radio propagation model $^{1}$ & Simple path loss model with log-normal shadowing effect \\
Minimum bit error rate $^{2}$ & $1 \times 10^{-8}$ \\
Radio transmission power & $-3 \mathrm{dBm}$ \\
\hline
\end{tabular}

1. An alpha value of 2.5, and a standard deviation of 6 with mean attenuation of 0 are used for the simple path loss and log-normal models respectively [48].

2. Based on the IEEE 802.15.4 model provided by the MiXiM framework [48], a transmission power of $-3 \mathrm{dBm}$ allows all the streetlights to communicate with their adjacent neighbours with a $99.9 \%$ successful packet delivery rate. 
During simulations, the necessary information for TALiSMaN's operation is communicated using a 19 byte packet: 4 bytes for the packet source and destination address, 1 byte for the message type coding, message versioning, command coding, command data length and data, 4 bytes for the packet timestamp, and 2 bytes for the packet checksum. Given that the travel direction of the detected road users is not available to TALiSMaN, and to allow sensor nodes to create the lighting conditions that offer optimum streetlight utility collaboratively, information on detected road users is relayed to any sensor node within a $150 \mathrm{~m}$ radius of the source node. However, this requires a network protocol to govern end-to-end information routing at the network layer of the WSN. As information on the detected road users is delay-sensitive, retransmission or guaranteed delivery of this information is not the focus of this protocol, but it should allow propagation of the information to all the sensor nodes within the confined distance with minimum delay. Therefore, a flooding protocol is adopted in our simulations. Nevertheless, uncontrolled flooding can lead to unnecessary network congestion and increased delays, which would consequently affect the performance of TALiSMaN. Therefore, the flood is constrained to discard packets once they have reached a distance of $150 \mathrm{~m}$ from the source node. During simulations, propagation errors and packet collision are introduced which cause packets to be discarded prematurely (see [49] for the details of radio propagation errors and packet collision).

To prevent the WSN from generating and forwarding redundant information, nodes only propagate the streetlight's operational state when a road user is detected. After a detection, nodes continually report their operational state to neighbouring streetlights at rate of $2 \mathrm{~Hz}$, while the road user continues to be within its sensing range. $2 \mathrm{~Hz}$ was chosen based on the simulation scenario considered, i.e. a 30 m average distance between two adjacent streetlights and a residential road with a speed limit of $30 \mathrm{mph}(48 \mathrm{~km} / \mathrm{h})$; therefore allowing each passing road user to be detected at least twice. Due to the routing protocol adopted (packet flooding) and the high network congestion that results (packet loss of between 23 $29 \%$ was experienced during simulations), nodes are programmed to continue to generate and forward packets for an additional 15 seconds after a road user is detected within its the sensing range. This extra forwarding mechanism increases the probability of streetlights receiving the latest operational state from the streetlights; as shown in the results below, this can be seen to deliver streetlight utility to all road users of greater than $90 \%$ (see Figure $8 \mathrm{a}$ ).

\section{Simulation Results}

In this section the performance of each lighting scheme is presented in terms of the streetlight utility experienced by simulated pedestrians and motorists, and the total energy consumed by 112 streetlights over a week (5 days for weekday traffic and 2 days for weekend traffic). 


\subsection{Streetlight Utility Experienced by Road Users}

To illustrate the behaviour of TALiSMaN, Figure 6 shows the lighting conditions of a road segment at different times $t$ as a result of a simulated pedestrian travelling towards streetlight $s_{12}$. At $t=2$, the presence of the pedestrian is detected by streetlight $s_{8}$ and this information is shared between neighbouring streetlights, i.e. $s_{3}$ to $s_{12}$. Upon receiving the information, the brightness levels of these streetlights are adjusted to create the lighting pattern needed by the pedestrian. As the pedestrian travels towards streetlight $s_{12}$, these optimum lighting conditions are shifted to the right along with presence detected by streetlight $s_{9}$ to $s_{12}$. Under such lighting conditions, TALiSMaN offered near-optimal streetlight utility to the pedestrian.

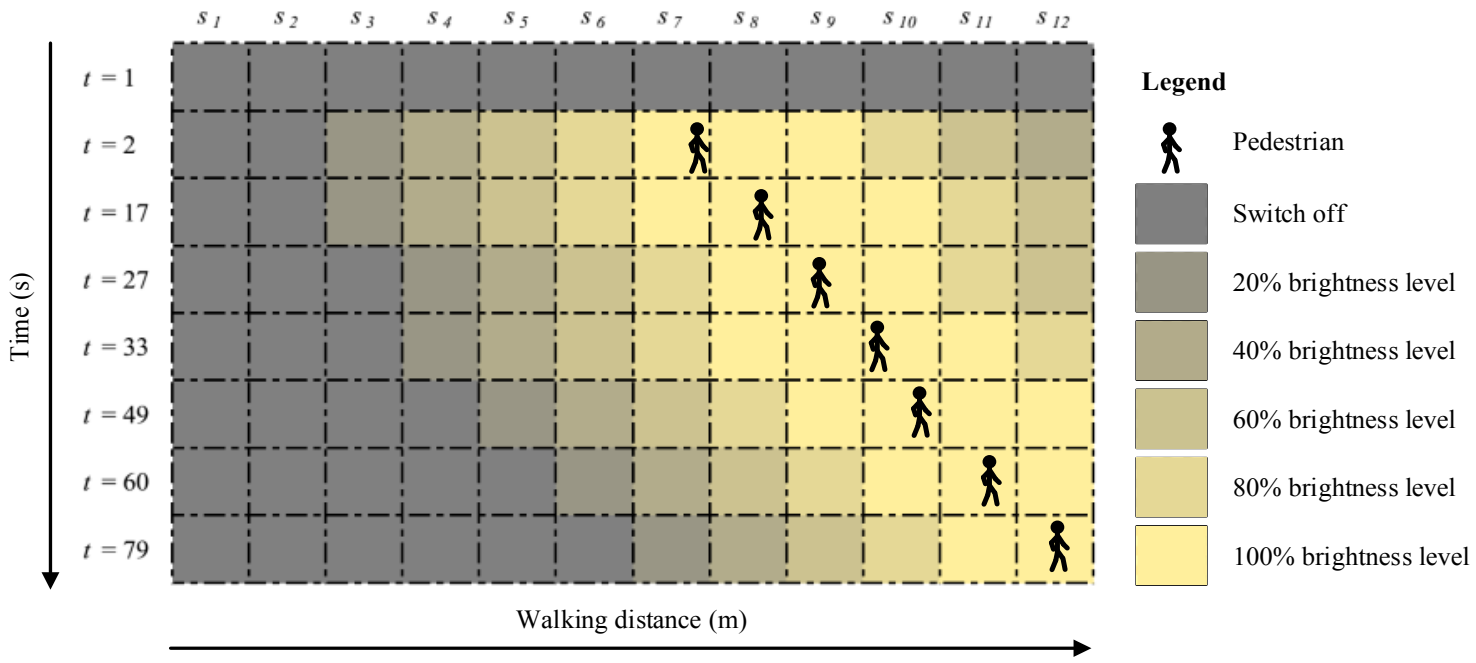

Figure 6. The dynamics of lit road segments (from top view) when a pedestrian travels from left to right.

Figure 7 shows the power output of a streetlight while operating TALiSMaN. During early operational hours, the power output of the streetlight is consistently $25 \mathrm{~W}$. This trend is due to the near-continuous stream of road traffic during these 'rush hours' preventing streetlights from switching off. The same trend can be observed as it approaches the morning rush hour. The streetlight is mostly switched off between midnight and the early morning as road traffic is lower compared to other operational hours. Although the streetlight is mostly switched off during these hours, TALiSMaN still retains the near-optimal usefulness of lighting to its users, as shown in Figure 8 (g).

Figure 8 shows the distribution of average streetlight utility experienced by each simulated road user from 16:00 to 08:00 the next day, when streetlights operate using the different lighting schemes. From the simulation results, none of the evaluated lighting schemes are able to offer perfect streetlight utility $(100 \%)$ in any of the streetlight operational hours for both the simulated pedestrians and motorists. This is even the case for streetlights that are always switched on at $100 \%$ illuminance output while operating the Conventional lighting scheme. This result, however, is expected as some of the road 
segments between two adjacent streetlights are larger than streetlight beam pattern $(30 \mathrm{~m})$ can cover. Owing to this, there are small sections of unlit road that have prevented perfect streetlight utility being achieved. In general, the streetlight utility experienced by the simulated motorists is higher than those experienced by the simulated pedestrians. This is due to StreetlightSim's placement of streetlights at the centre of roads, and their circular beam pattern. Therefore, simulated pedestrians encounter larger unlit road sections compared to simulated motorists.

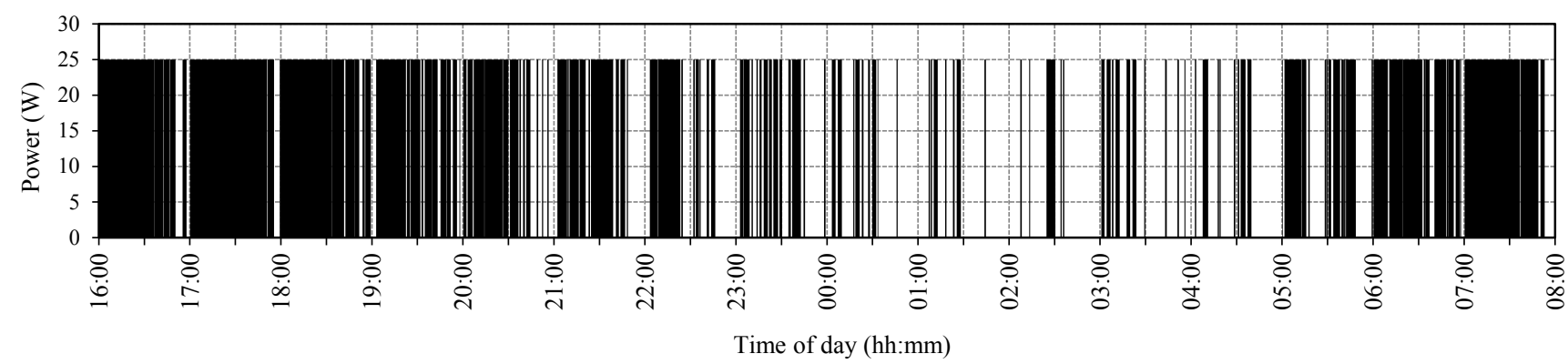

Figure 7. Power output modulation of a streetlight $(25 \mathrm{~W})$ during operational hours from 16:00 until 08:00 the next day while TALiSMaN is evaluated with traffic volume of 438 vehicles per day.

It can be seen that all simulated road users experience at least $90 \%$ utility during operational hours when using the Conventional lighting scheme. Similar trends can be observed when TALiSMaN is in use. Although Zoning has a similar performance in terms of pedestrian utility, motorist utility is severely impacted (this is to be expected, as it is not designed for these users). While motorists are not tracked by the scheme, their experienced utility was evaluated based on the lighting intended for pedestrians. Thus, the utility experienced by motorists is not consistent across streetlight operational hours (it varies between $0-95 \%$ ), and reduces when pedestrian traffic is low, i.e. during early morning. For Multi-sensor, all streetlights are always at $40 \%$ illuminance, and this is increased to $70 \%$ and $100 \%$ illuminance output when a motorist or pedestrian is $20 \mathrm{~m}$ and $10 \mathrm{~m}$ away respectively. Thus, it is unable to create the lighting conditions that can satisfy both the requirements of the pedestrians and the motorists, and has a reduced utility when compared to the Conventional and TALiSMaN lighting schemes. The increased traffic volume between the hours of 16:00 - 20:00 and 06:00 - 08:00 causes streetlights to constantly switch on at higher illuminance output, and hence higher utility can be observed at these times.

Philips Chronosense, Part-night and Dynadimmer are considered to be time-based dimming lighting schemes, as their illuminance is regulated according to predefined timetables. Owing to this, streetlight utility experienced by simulated road users also fluctuates according to their predefined timetable. For Part-night, all streetlights are switched on with $100 \%$ illuminance output except between midnight and early morning when they are switched off completely. Thus, Part-night has a similar utility to Conventional and TALiSMaN lighting schemes except during this period. 

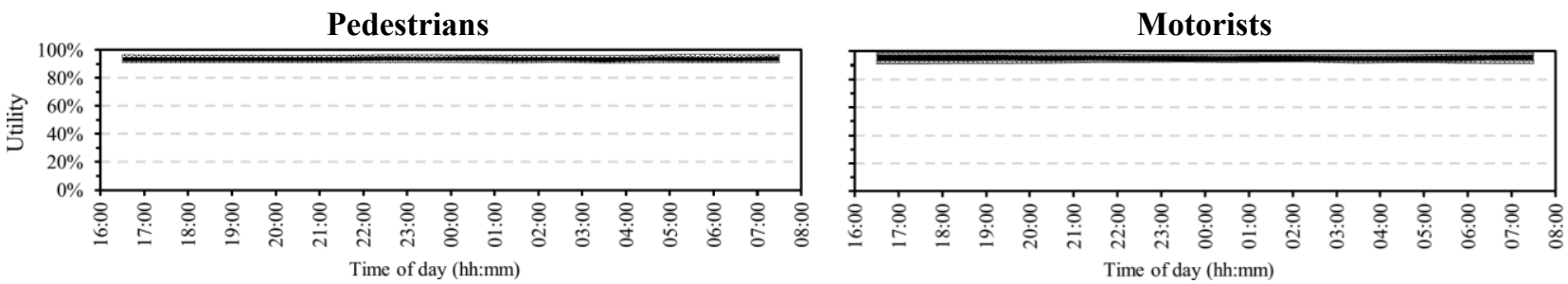

(a) Conventional
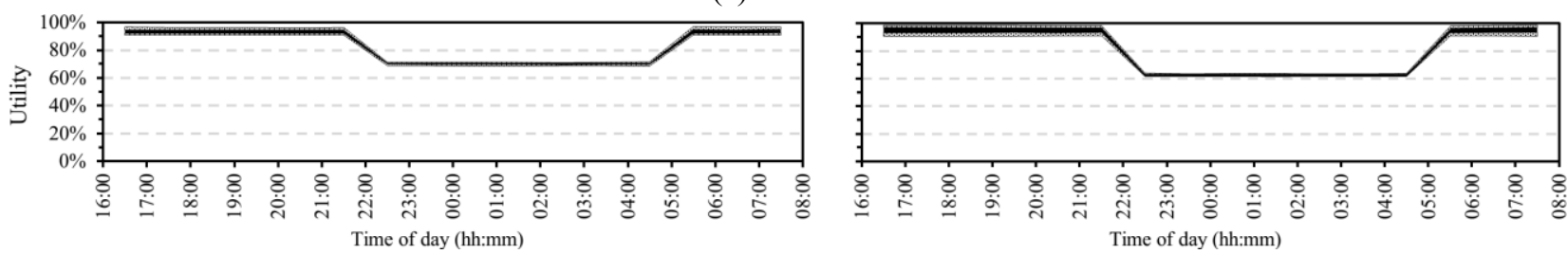

(b) Chronosense
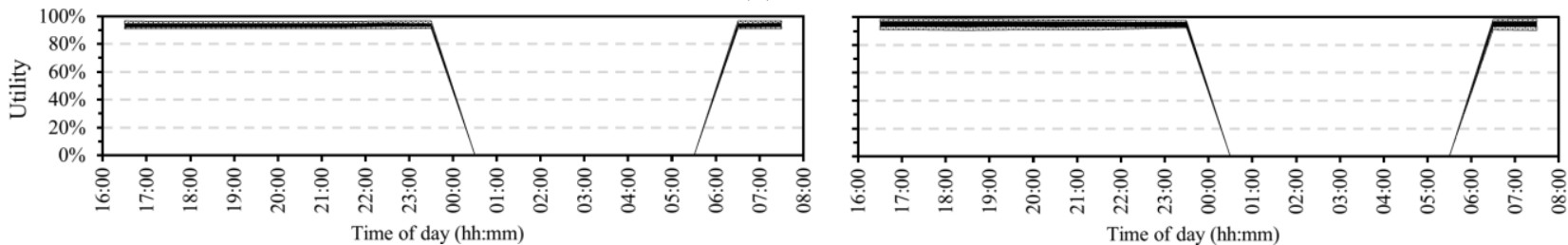

(c) Part-night
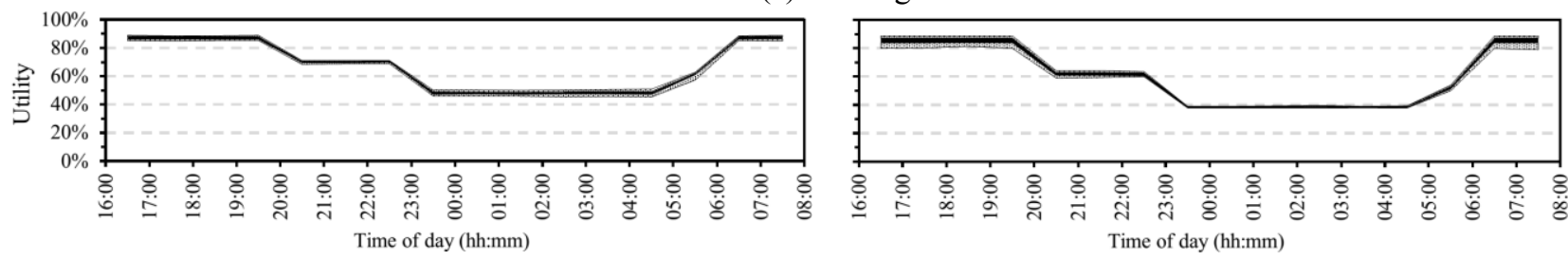

(d) Dynadimmer
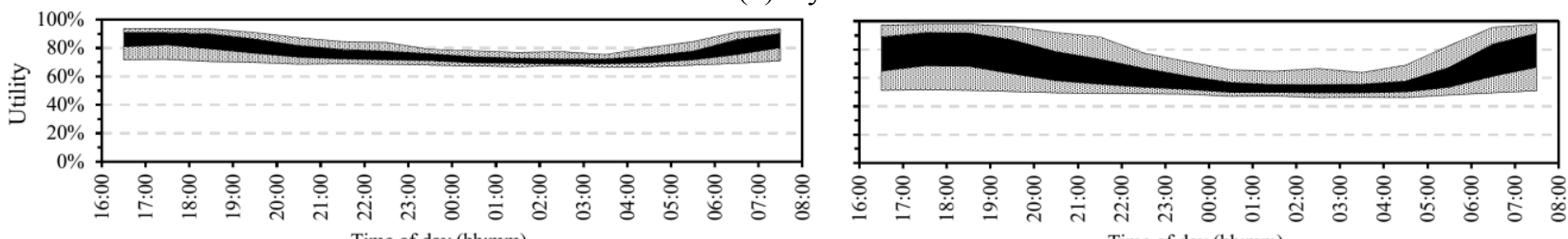

(e) Multi-sensor
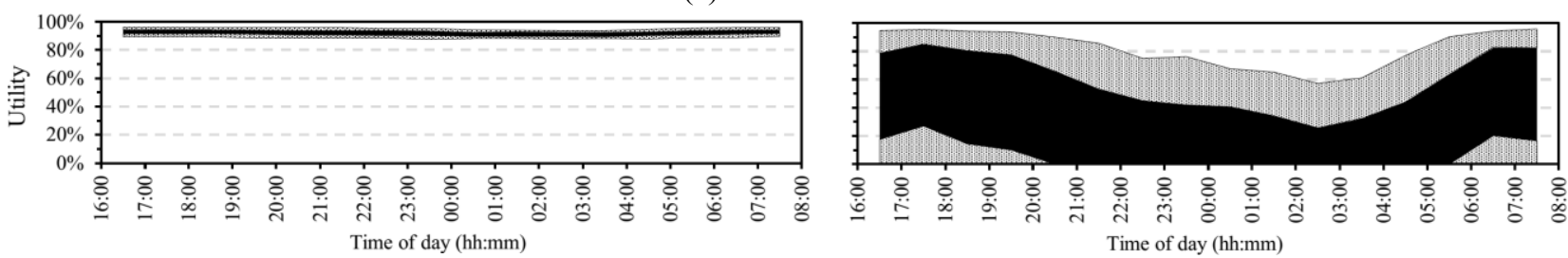

(f) Zoning
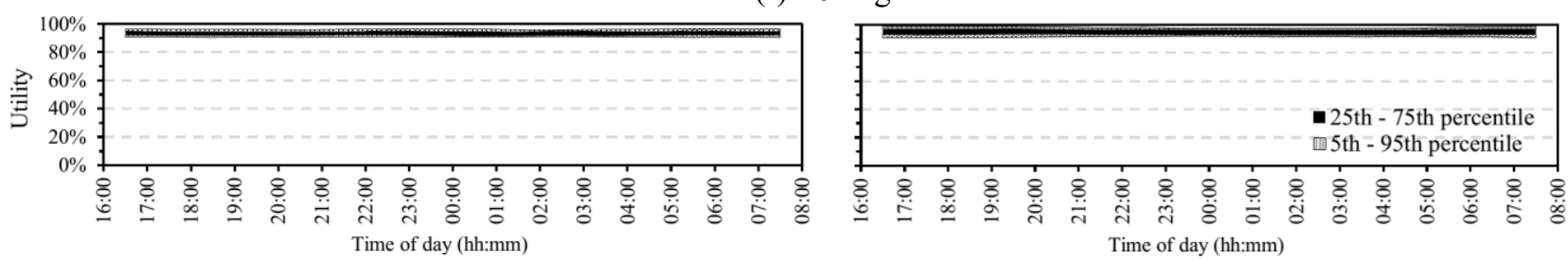

(g) TALiSMaN

Figure 8. Streetlight utility experienced by pedestrians (left) and motorists (right) during different streetlight operational hours with two percentile bands (inner band from 25 th to 75 th percentile, outer band from 5 th to 95 th percentile) with streetlights operating different lighting schemes. 
Philips Chronosense allows illuminance to be reduced to a predefined value at a predefined time. In our simulations, we consider this to be reduced to $65 \%$ between the hours of 22:00 and 05:00 (a typical setting of Philips Chronosense [8]). Correspondingly, the utility provided during these hours is also reduced to $68 \%-72 \%$ and $60 \%-65 \%$ for the simulated pedestrians and motorists respectively. For the remaining hours, this scheme offers similar utility to the Conventional and TALiSMaN lighting schemes. Compared to Philips Chronosense, Philips Dynadimmer allows multiple predefined illuminance output as summarised in Table 2. As shown Figure 8 (d), streetlight utility experienced by the simulated pedestrians and motorists varies according to five different streetlight operational periods. As the maximum illuminance output of the lighting scheme is set at $90 \%$, none of the simulated pedestrians or motorists are able to experience streetlight utility above $90 \%$ as demonstrated by other lighting schemes evaluated in this paper.

\subsection{Energy Consumption of Various Street Lighting Schemes}

To evaluate the energy demand of various street lighting schemes, the energy model shown in Eq. (11) is developed, where $e(N)$ is the energy consumed by a streetlight after $N$ discrete timesteps, $\varphi$ is the illuminance output of the streetlight $(\%), P_{\max }$ is the maximum power rating of the light source $(\mathrm{W})$, and $T$ is the duration of a single timestep $n$.

$$
e(N)=\sum_{n=0}^{N} P_{\max } \varphi T
$$

This model assumes that the streetlight energy consumption is directly proportional to its illuminance output, e.g. when the streetlight illuminance output is reduced to $80 \%$, the streetlight energy consumption is also reduced to $80 \%$ based on its maximum power rating, $P_{\max }$.

Figure 9 shows the total energy consumption of 112 streetlights in one week for different traffic volumes while operating different lighting schemes. The results show that the energy consumption of time-based dimming schemes, i.e. Conventional, Chronosense, Part-night and Dynadimmer is not dependent on traffic volume. Thus, their energy consumption remains constant while evaluated with different traffic volumes. As expected, Conventional is the least energy efficient of the lighting schemes considered. This is expected since the streetlights are always switched on at $100 \%$ illuminance. Chronosense, Part-night and Dynadimmer reduce illuminance at specific hours, hence reducing the energy consumption by $15 \%, 34 \%$ and $37 \%$ respectively, compared to Conventional. 


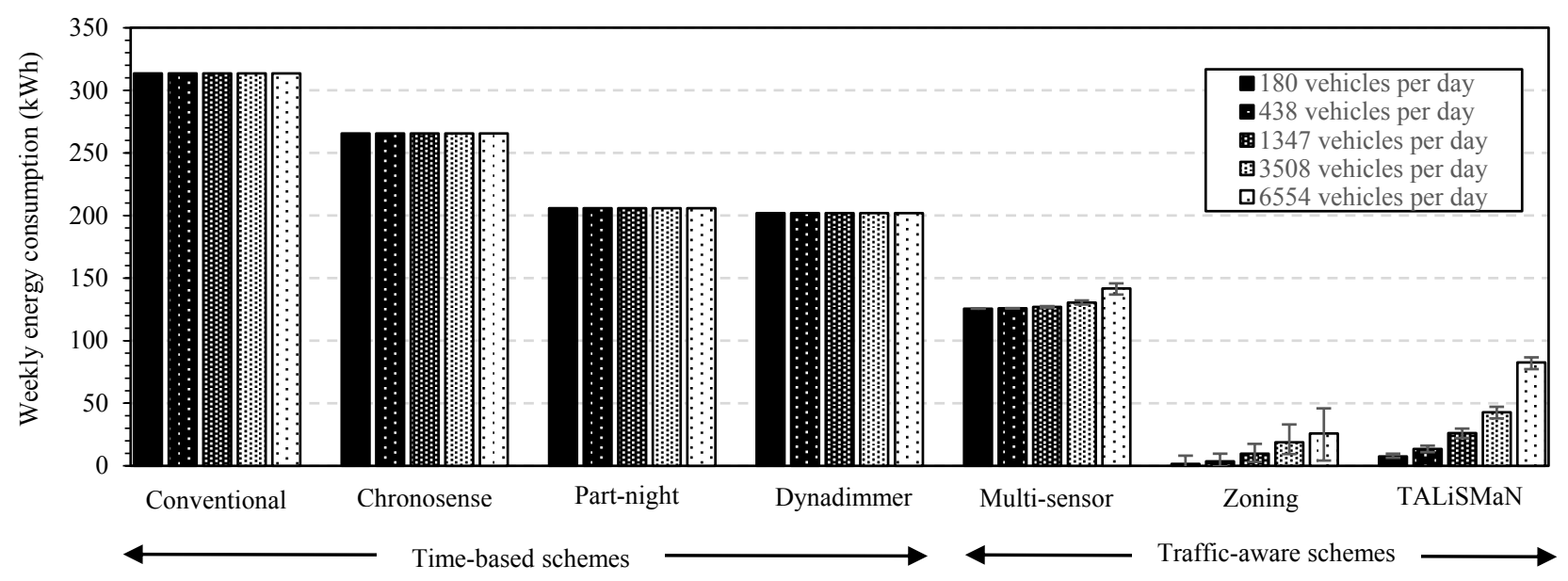

Figure 9. Mean weekly energy consumption of 112 streetlights while operating various street lighting schemes from 16:00 to 08:00 next day. The error bars represent the maximum and minimum energy consumption over ten repeated simulations.

In general, the energy consumption of the streetlights when operating traffic-aware lighting schemes, i.e. Multisensor, Zoning and TALiSMaN, increases with larger traffic volumes. This trend, however, is anticipated since these lighting schemes are designed to save energy by turning off or dimming lights when less traffic is present. As a result of increasing traffic volumes, a near-continuous stream of traffic is developed within the detection range of each sensor. Hence, the time each streetlight spends active is also prolonged, and thus energy consumption is also increased. The effect of increasing traffic volumes to Multi-sensor, however, is less pronounced compared to other traffic-aware lighting schemes. This is due to the streetlights operating Multi-sensor being constantly switched on at $40 \%$ illuminance output even when a road user is not detected.

Amongst all the evaluated lighting schemes, Zoning consumes the least energy for all the scenarios but its energy was expended for only a small proportion of road users, i.e. the pedestrians (14\%). This is because it was originally designed to address the needs of pedestrians. Owing to this, Zoning failed to provide a streetlight utility comparable to that of other schemes (see Figure 8). TALiSMaN required almost 3x more energy compared to Zoning. This result is justifiable because TALiSMaN aims to provide optimum lighting conditions that fulfil different road users' needs for street lighting. While Zoning may have application to areas with pedestrian-only traffic, for example some commercial areas and parks, it is clearly not applicable to those that have a mix of pedestrians and motorists. Furthermore, while Zoning requires pedestrians to carry GPS-enabled smartphones, TALiSMaN provides reasonable energy savings without any such demands. Therefore, the adoption of TALiSMaN in residential areas is more viable when compared to Zoning because traffic in such places normally comprises both pedestrians and motorists. Therefore, disregarding Zoning, TALiSMaN can be seen to consume between 2-55\% (depending on traffic volume) of the energy required by Multi-Sensor, the best-performing state-of-the-art 
technique. Furthermore, compared to conventional (or 'always-on') lighting scheme, TALiSMaN only consumes $1-2 \%$ of the energy.

Both Zoning and TALiSMaN exchange information between streetlights via a radio communication network. To evaluate the worst-case energy overhead of this, we consider that each streetlight has an IEEE 802.15.4 transceiver which is active for up to 16 hours per day, consuming $100 \mathrm{~mW}$ for both data transmission and reception [50]. This represents an overhead of $1.25 \mathrm{kWh}$ per week for both schemes, which is only $2-3 \%$ of the $40-65 \mathrm{kWh}$ of energy saved by TALiSMaN compared against the Multi-sensor lighting scheme.

Figure 10 shows the weekly energy consumption of various lighting schemes according to seasonal change in different months of year while evaluated with a traffic volume of 6554 vehicles per day. The operational hours of the streetlights are based on average sunset and sunrise times in Southampton, UK [51]. As the energy consumption of the streetlights is partially influenced by duration of streetlight operation, all the lighting schemes exhibit a trend with summer months having the lowest energy consumption over a year, and reach their peak during winter months. The length of daytime (i.e. when the streetlights are not switched on) is typically longer in summer months than winter months, hence shortening the required operational hours of the streetlights. In addition, road traffic during these hours is also expected to be lower (see Figure 5). Owing to these, energy consumption during these months is significantly lower compared to other months. Although energy consumption is reduced during summer months, traffic-aware lighting schemes still outperform comparable timebased lighting schemes.

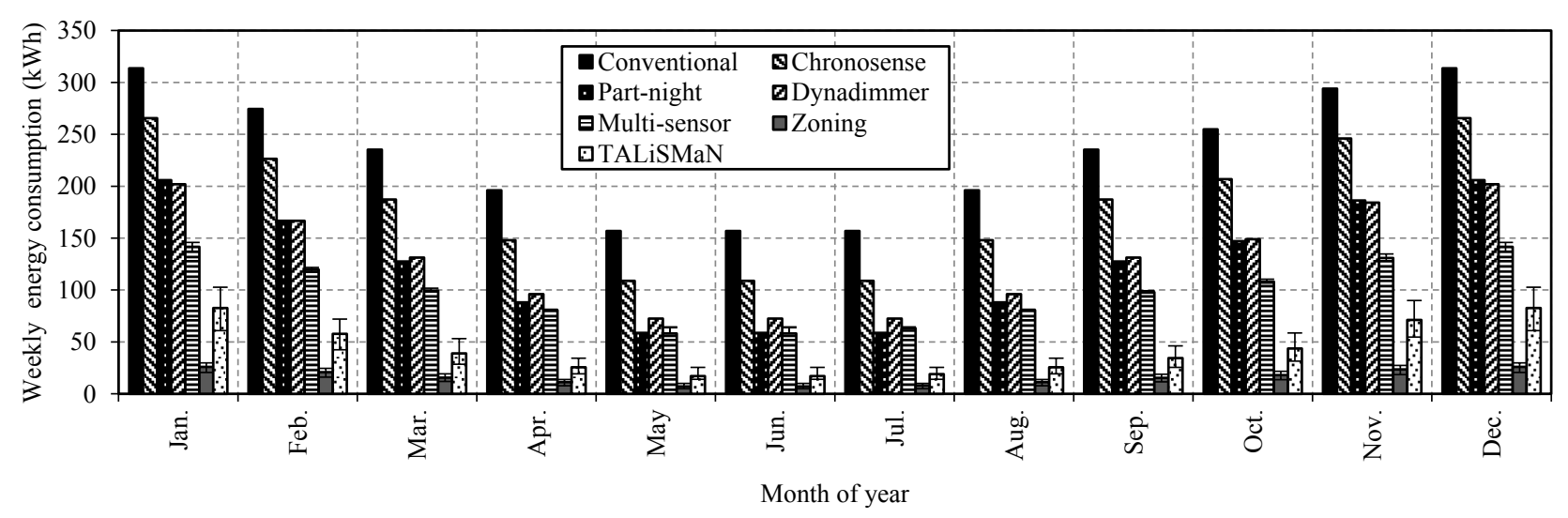

Figure 10. Weekly energy consumption of 112 streetlights for different months of year. The error bars represent the maximum and the minimum energy consumption for Multi-sensor, Zoning, and TALiSMaN lighting schemes over 10 repeated simulations while with traffic volume of 6554 vehicles per day. 


\section{Conclusions}

In this paper, we proposed a distributed Traffic-Aware Lighting Scheme Management Network (TALiSMaN) to create lighting conditions that maximise the utility of the streetlights, and improve their energy efficiency by minimising their energy use. TALiSMaN was simulated and its performance analysed and compared with state-of-the-art lighting schemes. We demonstrate that streetlights using TALiSMaN have a lower energy consumption than existing schemes, while offering comparable utility to conventional (or 'always-on') lighting. Based on the simulation results, TALiSMaN provides an energy saving of $45-98 \%$ (depending on traffic volume) compared to the state-of-the-art schemes evaluated. While the Zoning lighting scheme has a relatively low energy consumption, it typically cannot offer comparable streetlight utility to TALiSMaN. Our future work is currently investigating (1) novel communication protocols which are better suited to TALiSMaN's network architecture and traffic patterns, and (2) the extension of TALiSMaN to consider energymanagement for off-grid streetlights; these have become popular in areas where access to mains power is restricted.

\section{References}

[1] International Energy Agency, Light's Labour's Lost: Policies for Energy-efficient Lighting, France: IEA, 2006.

[2] K. Pease, “A Review of Street Lighting Evaluations: Crime Reduction Effects,” Surveillance of Public Space: CCTV, Street Lighting and Crime Prevention, vol. 10, pp. 47-76, 1999.

[3] P. O. Wanvik, "Effects of Road Lighting: An Analysis Based on Dutch Accident Statistics 1987-2006," Accident Analysis \& Prevention, vol. 41, no. 1, pp. 123-128, January 2009.

[4] S. Atkins S. Husain and A. Storey, The Influence of Street Lighting on Crime and Fear of Crime, United Kingdom: Home Office Crime Prevention Unit, 1991.

[5] M. Ramsay, The Effect of Better Street Lighting on Crime and Fear: A Review, United Kingdom: Home Office Crime Prevention Unit, 1991.

[6] S. Pellicer, G. Santa, A. L. Bleda, R. Maestre, A. J., Jara and A. Gomez Skarmeta, “A Global Perspective of Smart Cities: A Survey,” in Proc. 7th Int. Conf. Innovative Mobile and Internet Services in Ubiquitous Computing (IMIS), Taichung, 2013, pp. 439-444.

[7] Northeast Group, LLC, Global LED and Smart Street Lighting: Market Forecast (2014 - 2025), Washington: Northeast Group, LLC, 2014.

[8] Koninklijke Philips Electronics N. V., "Different Ways: The Dynadimmer and the Chronosense," 2010. [Online]. Available: http://www.lighting.philips.co.uk/subsites/oem/product_pages/dynadimmer_overview.wpd [Accessed: 21 May 2012].

[9] Warwickshire County Council, "Part Night Lighting”. [Online]. Available: http://www.warwickshire.gov.uk/partnightlighting [Accessed: 11 Nov. 2013].

[10] A. Mayeur, R. Brémond and J. Bastien, "The Effect of the Driving Activity on Target Detection as A Function of the Visibility Level: Implications for Road Lighting," Transportation Research Part F, vol. 13, pp. 115-128, 2010.

[11] S. I. Hong, H. S. Ryu, D. H. Yoon, C. G. In, J. C. Park and C. H. Lin, “A Development of LED-IT-Sensor Integration Streetlight Management System on Ad-hoc," in Proc. IEEE Region 10 Conf., Bali, 2011, pp 1331-1335.

[12] A. Siddiqui, A. Ahmad, H. K. Yang and C. Lee, "ZigBee based energy efficient outdoor lighting control system," in Proc. 14th Int. Conf. Advanced Communication Technology, Pyeong Chang, 2012.

[13] J. Zhang, G. F. Qiao, G. M. Song, H. T. Sun and J. Ge, “Group decision making based autonomous control system for street lighting," Measurement, vol. 43, no. 1, pp. $108-116,2013$.

[14] C. Jing, D. Shu and D. Gu, "Design of Streetlight Monitoring and Control System Based on Wireless Sensor Networks," in Proc. 2nd IEEE Conf. on Industrial Electronics and Applications, Harbin, 2007.

[15] M. Kostic and L. Djokic, "Recommendations for energy efficient and visually acceptable street lighting,” Energy, vol. 34 , pp. 1565 - $1572,2009$.

[16] J. H. Sun, J. F. Su, G. S. Zhang, Y. Li and C. Zhao, “An Energy-saving Control Method Based on Multi-sensor System for Solar Street Lamp,” in Proc. Int. Conf. Digital Manufacturing and Automation (ICDMA), ChangSha, 2010, pp 192-194.

[17] Delft University of Technology, "Intelligent Street Lighting at TU Delft saves up to $80 \%$ on energy". [Online]. Available: http://home.tudelft.nl/en/current/latest-news/article/detail/intelligente-straatverlichting-tu-delft-kan-tot-80-energie-besparen/ [Accessed: 19 Mar. 2012].

[18] N. Zotos, C. Stergiopoulos, K. Anastasopoulos, G. Bogdos, E. Pallis and C. Skianis, "Case Study of A Dimmable Outdoor Lighting System with Intelligent Management and Remote Control," in Proc. Int. Conf. Telecommunications and Multimedia (TEMU), Chania, 2012 , pp. $43-48$.

[19] A. Lavric, V. Popa and I. Finis, “The Design of a Street Lighting Monitoring and Control System," in Proc. Int. Conf. and Exposition Electrical and Power Engineering, Iasi, Romania, 2012.

[20] F. Leccese, "Remote-Control System of High Efficiency and Intelligent Street Lighting Using a ZigBee Network of Devices and Sensor," IEEE Transactions on Power Delivery, vol. 28, no. 1, pp. $21-28,2013$. 
[21] E. Nefedov, M. Maksimainen, S. Sierla, C. W. Yang, P. Flikkema, I. Kosonen and T. Luttinen, "Energy efficient traffic-based street lighting automation," in Proc. IEEE 23rd Int. Symp. Industrial Electronics, Istanbul, 2014, pp. 1718 - 1723.

[22] Y. Wu, C. Shi and W. Yang, "Study of Acquisition Streetlights Background Signal by Multi-sensor Array," in Proc. Int. Conf. Control Automation and Systems (ICCAS), Gyeonggi-do, 2010, pp. 1000-1003.

[23] R. Müllner and A. Riener, "An Energy Efficient Pedestrian Aware Smart Street Lighting system," International Journal of Pervasive Computing and Communication, vol. 7, no. 2, pp. 147-161, 2011.

[24] Ofcom, “Communications Market Report 2012," 2012. [Online]. Available: http://stakeholders.ofcom.org.uk/binaries/research/ cmr/cmr12/CMR_UK_2012.pdf. [Accessed: 12 Sept. 2013].

[25] Z. Zhuang, K. H. Kim and J. P. Singh, "Improving energy efficiency of location sensing on smartphones.," in Proc. 8th Int. Conf. Mobile systems, applications, and services, 2010.

[26] A. Carroll and G. Heiser, "An Analysis of Power Consumption in a Smartphone," in Proc. USENIX annu. technical conference, 2010.

[27] S. P. Lau, G. V. Merrett and N. M. White, "Energy-Efficient Street Lighting through Embedded Adaptive Intelligence," in Proc. Int. Conf. Advanced Logistics and Transport, Tunisia, 2013, pp. $53-58$.

[28] S. P. Lau, G. V. Merrett and N. M. White, "StreetlightSim: A Simulation Environment to Evaluate Networked and Adaptive Street Lighting," in Proc. IEEE Asia Pacific Conf. Wireless and Mobile Technology, Bali, 2014.

[29] Y. Lin, W. Cheng, C. Wu and Y. Sun, "An Intelligent Lighting Control System Based on Ergonomic Research," in Proc. Int. Conf. Consumer Electronics, Communications and Networks (CECNet), XianNing, 2011, pp.4744-4747.

[30] P. Raynham, “An Examination of the Fundamentals of Road Lighting for Pedestrians and Drivers," Lighting Research and Technology, vol. 36, no. 4, pp. 307-316, 2004.

[31] N. Davoudian and P. Raynham, “What Do Pedestrians Look At At Night?” Lighting Research and Technology, vol. 44, no. 4, pp. 438-448, 2012.

[32] S. Fotios and C. Cheal, "Obstacle Detection: A Pilot Study Investigating the Effects of Lamp Type, Illuminance and Age,” Lighting Research and Technology, vol. 41, pp. 321 - 342, 2009.

[33] F. Fotios and C. Cheal, "Using Obstacle Detection to Identify Appropriate Illuminances for Lighting in Residential Roads," Lighting Research and Technology, vol. 45, pp. $362-376,2013$.

[34] T. Fujiyama, C. Childs, D. Boampong and N. Tyler, "Investigation of Lighting Levels for Pedestrians: Some Questions About Lighting Levels of Current Lighting Standards," in Proc. Int. Conf. Walking in the 21st Century, Zurich, 2005, pp.1-13

[35] British Standards Institution, BS EN 13201-2:2003 Road Lighting; performance Requirements, London: BSI, 2003.

[36] A. Haans and Y. A. de Kort, "Light Distribution in Dynamic Street Lighting: Two Experimental Studies on Its Effects on Perceived Safety, Prospect, Concealment, and Escape," Journal of Environmental Psychology, 2012

[37] L. K. Staplin, "Nightime Hazard Detection on Freeways under Alternative Reduced Lighting Conditions," in Human Factors and Ergonomics Society: 29th Annual Meeting, 1985.

[38] Ö. Güler and S. Onaygil, “The Effect of Luminance Uniformity on Visibility Level in Road Lighting,” Lighting Research and Technology, vol. 35, no. 3, pp. 199-215, 2003

[39] A. Mayeur, R. Brémond and J. M. C. Bastien, "Effects of the Viewing Context on Target Detection. Implications for Road Lighting Design," Applied Ergonomics, vol. 41, no. 3, pp. 461 - 468, 3 May 2010.

[40] R. Brémond, V. Bodard, E. Dumont and A. Nouailles-Mayeur, "Target Visibility Level and Detection Distance on A Driving Simulator," Lighting Research and Technology, pp. 1-14, 2012.

[41] D. A. Schreuder, Road Lighting for Safety, London: Thomas Telford Ltd., 1998

[42] S. P. Hoogendoorn and W. Daamen, "Free Speed Distributions for Pedestrian Traffic," Transportation Research Board: 85th Annual Meeting, 2006.

[43] S. P. Lau (2014), "StreetlightSim". [Online]. Available: www.streetlightsim.ecs.soton.ac.uk.

[44] United Kingdom. Department for Transport, Traffic dataset: Street-level traffic figures for all regions and local authorities (Minor roads), London: Department for Transport, 2011. [Online]. Available: http://data.dft.gov.uk/gb-traffic-matrix/AADF-data-minor-roads.xls [Accessed: 4 Jun. 2012]

[45] European Platform on Mobility Management, “TEMS - The EPOMM Modal Split Tool”. [Online]. Available: http://www.epomm.eu/tems [Accessed: 4 Jun. 2013].

[46] United Kingdom. Department for Transport, Traffic distribution by time of day on all roads in Great Britain, 2011, London: Department for Transport, 2011. [Online]. Available: https://www.gov.uk/government/uploads/system/uploads/attachment_data/file/316563/tra0308.xls [Accessed: 4 Jun. 2012]

[47] Wireless Medium Access Control (MAC) and Physical Layer (PHY) Specification for Low Rate Wireless Personal Area Networks, IEEE Std. 802.15.4, 2003.

[48] MiXiM (mixed simulator). [Online]. Available: http://mixim.sourceforge.net/index.html [Accessed: 20 Mar. 2012].

[49] A. Köpke, M. W. K. Swigulski, D. Willkomm, P. T. Haneveld Klein, T. E. V. Parker, O. W. Visser, H. S. Lichte and S. Valentin, "Simulating wireless and mobile networks in OMNeT++ the MiXiM vision," in 1st international conference on Simulation tools and techniques for communications, networks and systems \& workshops, Brussels, Belgium, 2008.

[50] Texas Instruments, Data sheet: A True System-on-Chip Solution for 2.4 GHz IEEE 802.15.4 and ZigBee Application, 2011. [Online]. Available: http://www.ti.com/lit/ds/swrs081b/swrs081b.pdf. [Accessed: 05 Feb. 2015].

[51] UK Hydrographic Office, "Her Majesty's Nautical Almanac Office," 2014. [Online]. Available: http://astro.ukho.gov.uk/surfbin/first_beta.cgi. [Accessed: 23 Jul. 2014]. 
Sei Ping Lau received the BSc degree (Hons) in Information Technology from Universiti Malaysia Sarawak and the MSc degree from Universiti Teknologi Malaysia, in 2001 and 2003 respectively. Currently, he pursuing his PhD at University of Southampton, UK.

Geoff V. Merrett received the BEng degree (Hons) in Electronic Engineering (2004) and the PhD degree (2009) from the University of Southampton, UK where he is currently an Associate Professor. He has authored over 80 papers, is a member of the IEEE and IET, and was General Chair of the ENSsys workshops in 2013 and 2014.

Alex S. Weddell received the MEng (Hons) in Electronic Engineering (2005) followed by a $\mathrm{PhD}$ (2010) from the University of Southampton. He was appointed as a Lecturer in 2013. He has authored over 25 papers, and has special interests in the areas of energy-aware systems and energy harvesting.

Neil White obtained a PhD from the University of Southampton in 1988 for a thesis describing the piezoresistive effect in thick-film resistors. He is a Chartered Engineer, Fellow of the IET, Senior Member of the IEEE, Fellow of the IoP and a Chartered Physicist. His research interests include thick-film sensors, intelligent instrumentation, MEMS, self-powered microsensors and sensor networks. 